\title{
Management of hypoglycemia after gastric bypass: a difficult challenge
}

Silva-Fernández, J; García-Manzanares, A; Gómez-Alfonso, FJ, López-Iglesias, M; Gómez-García, I Endocrinology and Nutrition Section. Macha Centro General Hospital. Alcázar de San Juan (Spain).

\section{Introduction}

Postprandial hypoglucemia with hyperinsulinism after laparoscopic gastric bypass is an uncommon complication with an estimated prevalence of $0.2 \%$. Its treatment includes acarbose, diazoxide, somatostatin and GLP-1 analogues or calcium channel blockers in case a strict diet does not work.

\section{Case report}

A 42-year-old woman with history of gastric bypass in 2005 due to severe obesity (preoperative weight 150 $\mathrm{kg}$ ) attended our clinic 3 years after surgery reporting hypoglycemic episodes occasionally accompanied by unconsciousness, occurring 3 hours after food intake. The episodes resolved after sugar intake.



ACTH stimulation test ruled out adrenal insufficiency. findings.

A glucose tolerance test was also performed showing hypoglycemia and hyperinsulinism.

A CT scan of the abdomen did not find pancreatic nodules.

\begin{tabular}{l} 
Sequence of treatments \\
Fractionated diet \\
Acarbose \\
Lanreotide \\
Diazoxide \\
\hline Corporocaudal pancreatectomy \\
Liraglutide \\
Total pancreatectomy \\
no hypoglycemic episodes were \\
the patient developed diabetes \\
with insuline. \\
ncreatectomy the patient had a \\
emisodes even after insuline \\
aload test with mixed meal showed \\
\hline 24 has prescribed but we had to
\end{tabular}

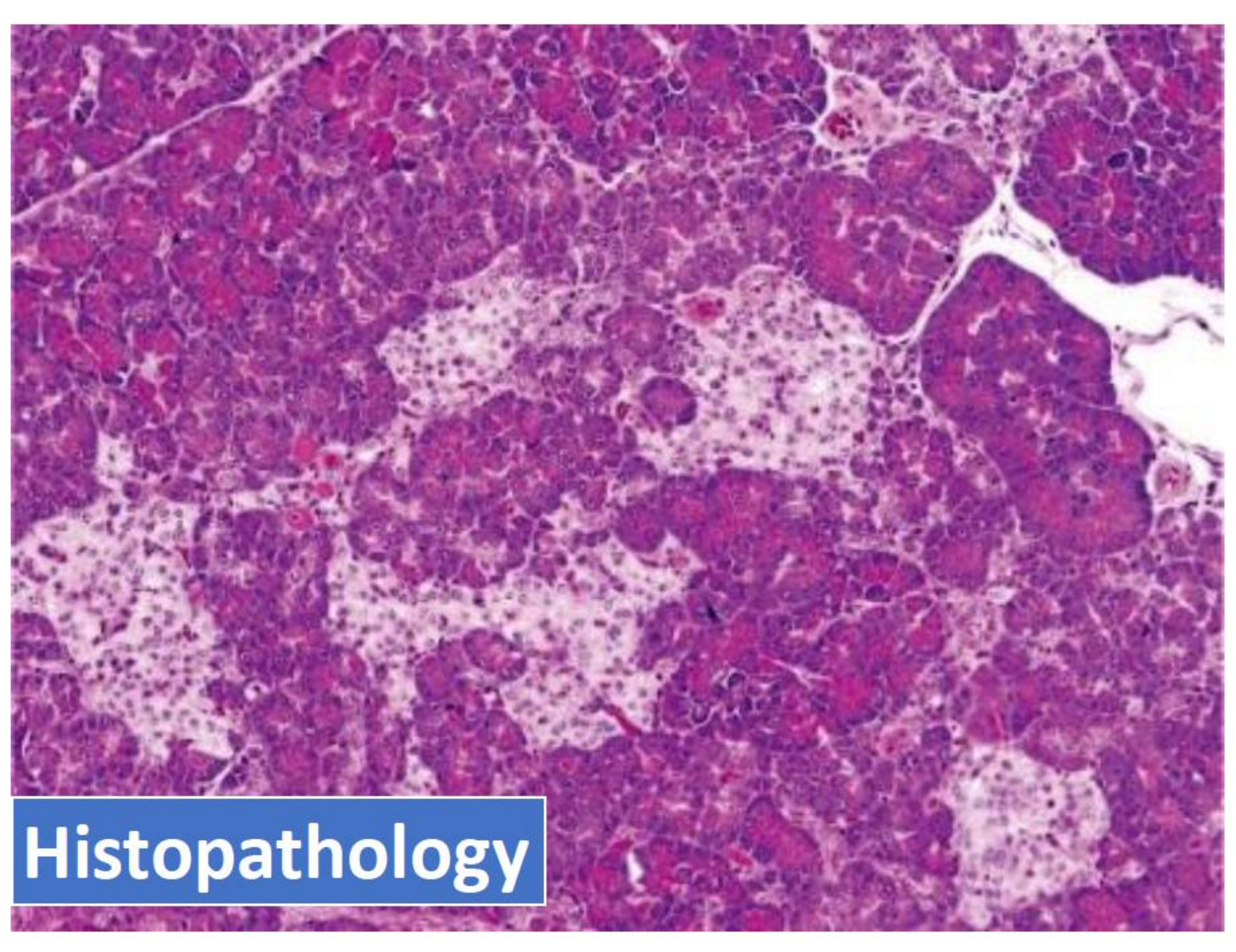

\section{Conclusions}

There is no treatment of choice for patients with nesidioblastosis. Medical treatment, including somatostatin and GLP-1 analogues, should be tried before surgery. When medical treatment fails, subtotal pancreatectomy is a good option to control hypoglucemia and preserve pancreatic function. When hypoglycemia persists, total pancreatectomy should be considered. 